\title{
BMJ Open Protocol for a randomised, placebo- controlled pilot study for assessing feasibility and efficacy of faecal microbiota transplantation in a paediatric ulcerative colitis population: PediFETCh trial
}

Nikhil Pai, ${ }^{1}$ Jelena Popov ${ }^{1,2}$

To cite: Pai N, Popov J. Protocol for a randomised, placebo-controlled pilot study for assessing feasibility and efficacy of faecal microbiota transplantation in a paediatric ulcerative colitis population: PediFETCh trial. BMJ Open 2017;7:e016698. doi:10.1136/ bmjopen-2017-016698

- Prepublication history for this paper is available online. To view these files please visit the journal online (http://dx.doi. org/10.1136/bmjopen-2017016698).

Received 8 March 2017

Revised 17 June 2017

Accepted 13 July 2017

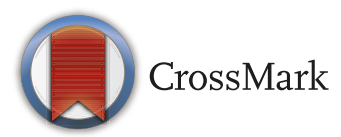

${ }^{1}$ Division of Pediatric Gastroenterology \& Nutrition, Department of Pediatrics, McMaster University, Hamilton, Canada

${ }^{2}$ McMaster Integrative Neuroscience Discovery and Study Graduate Program, McMaster University, Hamilton, Canada

Correspondence to

Dr. Nikhil Pai;

pain@mcmaster.ca

\section{ABSTRACT}

Introduction Ulcerative colitis (UC) is a chronic, relapsing condition characterised by colonic inflammation. Increasing prevalence in early-age diagnosis provides opportunities for additional complications in later life as a result of prolonged exposure to inflammatory and therapeutic insults, necessitating novel avenues for therapeutics which may result in fewer side effects. Faecal microbiota transplantation (FMT) has previously demonstrated potential therapeutic benefit in an adult randomised-controlled trial and several recurrent Clostridium difficile infection studies. This phase Ib pilot will be the first randomised, single-blinded, placebocontrolled trial to assess feasibility and patient outcomes in a paediatric inflammatory bowel disease (IBD) population. Methods and analysis Fifty patients will be randomised $1: 1$ to receive normal saline control or active sample. Enema administrations will be performed two times per week for 6 weeks, followed at a 6 -month follow-up period. Feasibility outcomes will include measures of patient eligibility, recruitment, willingness to participate, samples collections, hospitalizations and drop-out rate. Improvements in disease symptoms will determine the efficacy of treatment. Clinical disease scores will be taken throughout the study period using the Paediatric Ulcerative Colitis Activity Index (PUCAI). Monitoring of inflammatory markers in blood and stool will be performed at regular intervals. Microbiome analysis will be conducted on stool samples collected throughout the trials period. Imaging and endoscopic surveillance will be conducted if clinically necessary.

Ethics and dissemination Ethics was obtained from local hospital research ethics boards across all three sites. Health Canada and FDA approval was obtained for the use of an Investigatory New Drug product. Results from this trial will be presented in international conferences and published in peerreview journals.

Trial registration number Trial registration number: NCT02487238; preresults.

\section{INTRODUCTION}

Ulcerative colitis (UC) is a disease characterised by chronic inflammation of the colonic
Strengths and limitations of this study

- This multicentre pilot study is the first randomised, placebo-controlled trial assessing the feasibility and clinical efficacy of a faecal microbiota transplantation protocol for the treatment of paediatric ulcerative colitis and inflammatory bowel disease-unclassified (IBD-U). This pilot trial will provide insight towards the role of FMT in the treatment of paediatric inflammatory bowel disease (IBD).

- Results from this trial will be valuable for future faecal microbiota transplantation research involving larger population sizes, through multicentre, doubleblinded IBD trials.

- The lack of investigator blinding (single-blinding) is a limitation to the study design.

- The strengths and limitations of normal saline versus autologous stool as placebo are unclear, with previous investigators having used both in studies of FMT for recurrent Clostridium difficile colitis and FMT for the treatment of IBD.

mucosa. Approximately 104000 Canadians are affected by UC, and Canada has among the highest incidence in the world. ${ }^{1-3} \mathrm{~A}$ diagnosis of UC can be debilitating in childhood. Chronic diseases can have significant impacts on children, and UC may particularly affect childhood growth and development. ${ }^{45}$ Longterm immunosuppression is often required, and paediatric patients may face higher lifetime rates of lymphoma and infection given their longer duration of illness. While colectomy may be considered curative, prophylactic removal of the colon in childhood can have long-term impacts on fertility, and may negatively affect psychosocial function. ${ }^{67}$ For many paediatric UC patients, their quality of life is marred by flares of abdominal 
pain, bloody diarrhoea, and treatments that have significant toxicity. ${ }^{8}$

The traditional approach to managing UC involves immunosuppression to dampen an overactive immune system. ${ }^{8}$ An alternative strategy involves focusing on those factors that cause upregulation of the immune system in the first place. The gut microbiome is a provocative candidate. The intestinal tract is a rich immunologic organ. Numerous immune receptors (toll-like receptors and NOD-like receptors) interact with the intestinal lumen to directly respond to bacterial antigens and trigger systemic immune responses. ${ }^{9}$

Human biotherapy, or faecal microbiota transplantation (FMT), involves the administration of a stool and water mixture from a healthy screened donor. By modifying intestinal bacteria through FMT-effectively replacing it with a healthier bacterial milieu-we may be able to prevent the cascade of immune disruption that characterises UC.

\section{Rationale}

FMT has primarily been used for the treatment of recurrent Clostridium difficile colitis. A recent systematic review showed that over $90 \%$ of patients with recurrent $C$. difficile infection have been cured with FMT. ${ }^{10}$ These results have since been replicated in larger studies worldwide and strongly implicate colonic bacteria as a potential therapy for other gastrointestinal disease conditions. ${ }^{11} 12$

Four small case series have demonstrated success of FMT for paediatric inflammatory bowel disease (IBD) ${ }^{13-16}$ Protocols and response rates varied across each study, but lower gastrointestinal tract administration yielded clinical response rates in $67 \%-100 \%$ of patients. ${ }^{13} 14$

Two single-centre paediatric case reports have been recently published showing marked clinical improvement in two patients with severe colitis. A 2015 case report described a 18-month-old girl baby presenting with an early-onset colitis with UC-like phenotype, who responded after 7 serial FMT infusions with donor stool from and age-matched niece and older brother. ${ }^{17}$ A 2016 case report described an 11-year-old girl with corticosteroid-dependent UC who responded after serial FMT infusions every 2-4 weeks over a 10-month period. The patient remained in clinical remission at 40 weeks post final FMT and showed complete endoscopic healing. ${ }^{18}$ A further 2016 case report described a 3-year-old girl baby with acute severe UC who was refractory to aminosalicylates and all immunosuppressive drugs. ${ }^{19}$ She received 6 successive FMT enemas and 4 FMT via nasoduodenal tube over 10 days, but ultimately required colectomy. Donor faecal microbiota was not identified in the patient, and the authors concluded that due to the severely damaged colonic epithelium, paucity of crypts and overall decrease of mucous in the outer layer, the donor microbiota could not be retained by the recipient. The authors concluded that patients with mild disease might be a better candidate for FMT. However, factors that optimise bacterial transfer from donor to recipient in FMT remain unclear, and mechanistic conclusions from single-centre case reports remain speculative at this time. ${ }^{19}$

\section{Significance}

Factors that contribute to the success of FMT in the treatment of IBD remain unclear and need definitive randomised-controlled trials to identify benefit.

This pilot study will be the largest randomised, singleblind, placebo-controlled trial evaluating the role of FMT in paediatric UC ever conducted. This pilot study will built on the FMT in adult UC trial recently completed at our centre. ${ }^{20}$ We will assess the feasibility of delivering two times per week faecal enemas from anonymous faecal microbial donors, to patients with paediatric UC. Previous faecal transplant studies in paediatrics have typically used within-family, or within-household donors with varying frequencies of transplant delivery, most of which were fewer than the frequency proposed in this study. These differences in donor selection and transplant frequency may have compromised efficacy. This pilot will help identify factors associated with the success or failure of FMT in paediatric UC. Our results will provide important preliminary data for the design, completion of a definitive paediatric trial with a larger sample size and power calculation to attain statistical significance of our primary clinical outcomes in the future.

This study, and smaller paediatric trials by other investigators, suggests the following: FMT is an effective therapy in adult UC, FMT is an effective therapy for paediatric UC, donor selection has a significant role in optimising therapeutic response and there may be differences in response between patients with duration of disease $\leq 1$ year versus $>1$ year $^{20}$. For these reasons, a large randomised, singleblind, placebo-controlled trial in paediatric UC patients, who typically have a shorter disease duration, using an anonymous donor, will be scientifically and clinically valuable.

The aim of this paper was to describe the first randomised-controlled protocol for FMT in a paediatric IBD population.

\section{Study objectives}

The objective of this pilot study was to assess the feasibility of FMT for the therapy of paediatric UC. Specifically, we will test the hypothesis that a protocol of two times per week enemas over 6 weeks, using faecal transplant material from an anonymous unrelated donor, will improve the efficacy of FMT in paediatric UC patients, and build a framework for future studies to assess the effectiveness of FMT intervention. We will evaluate key feasibility measures of the following:

1. Participant recruitment (sample size)

2. Participant retention (sample size)

3. Participant eligibility criteria (sample size)

4. Acceptance of patients to participate in study (process)

5. Effect of intervention on disease primary and secondary disease outcomes (clinical) 
Assessed for eligibility:

Age: 3-17

Diagnosis: UC/IBD-U, PUCAI $\geq 15$,

elevated fecal calprotectin or C-reactive

protein

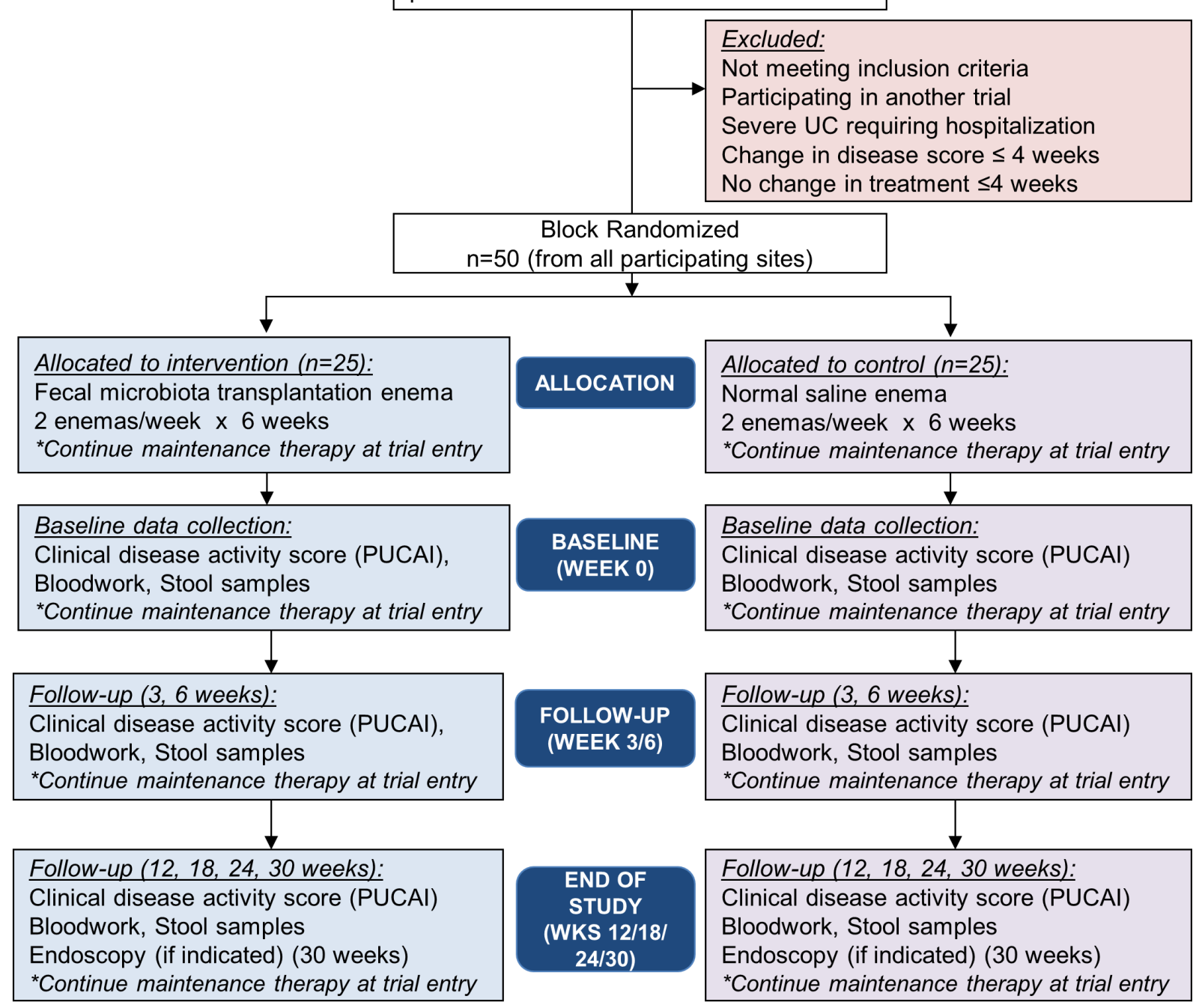

Figure 1 Study protocol. IBD-U,inflammatory bowel disease-unclassified; PUCAI, Paediatric Ulcerative Colitis Activity Index; UC, ulcerative colitis.

6. Effect of intervention on faecal bacterial community structure (clinical)

7. Rate of adverse events inpatients receiving FMT (clinical).

\section{METHODS AND ANALYSIS \\ Study design}

The PediFETCh Trial is a randomised, placebo-controlled multicentre trial. It uses a parallel arm approach consisting of a faecal transplantation group and a placebo group. In Ontario, Canada, there are two sites involved in this multicentre trial: McMaster Children's Hospital, Hamilton, and Children's Hospital of Western Ontario, London (primary and secondary sites, respectively).

Patients are seen on-site for all scheduled visits, in accordance with the protocol (figure 1). Faecal enemas are screened, prepared and tested by Rebiotix, and sent to the sites for patient administration. Enemas are administered at the respective site's paediatric gastroenterology clinics by study team members familiar with the singleblinded FMT enema protocol, two times per week for 6 weeks.

Patient enrolment began in November 2015 at McMaster Children's Hospital and is tentatively planned for Summer, 2017 at Children's Hospital of Western Ontario. A third site is projected to receive research ethics board approval in Fall, 2017 (Sainte Justine's Hospital, Montreal). We aim to complete recruitment of 50 patients with a target recruitment end-date of Fall, 2018. No other paediatric Gastroenterology sites in Canada are currently offering FMT trials for paediatric UC; thus, patients referred from outside centres for entry into our study will be considered if they meet eligibility criteria. 


\section{OUTCOME MEASURE ANALYSIS}

PUCAI; MRE: Magnetic Resonance Enterography

\section{Eligibility criteria}

Paediatric patients (aged 3-17 years) with UC and IBD-unclassified (IBD-U) subtype are eligible for the study. Patients classified as IBD-U are included because their distribution of disease is often limited to the large intestine-like classical UC. Eligible patients must also demonstrate signs of disease activity, as determined by measures of elevated inflammatory markers (specifically faecal calprotectin and C-reactive protein (CRP)), PUCAI scores of $\geq 15$ or increased disease activity supported by endoscopic findings. ${ }^{1321-23}$

Subjects are excluded if they are participating in another clinical trial, are unable to give informed consent or assent, have severe comorbid medical illness, have concomitant $C$. difficile infection or require hospitalisation (at discretion of the treating physician; typically, PUCAI $>65)$. Continued treatment with 5-ASA, azathioprine, 6-mercaptopurine or anti-TNF $\alpha$ therapy (eg, infliximab) will be permitted if taken at stable dose for $\geq 4$ weeks prior to randomization. No new medical therapies (eg, corticosteroids, antibiotics and probiotics) will be permitted during the study period, unless patients meet criteria for a suspected adverse event.

Patients younger than 3 years will also be excluded as these patients meet definition for 'Infantile IBD', which may have a different biological and clinical phenotype than other paediatric age presentations of IBD. ${ }^{2425}$

\section{Randomisation}

All patients seen at McMaster Children's Hospital's Division of Gastroenterology who meet eligibility criteria will be approached for participation in the study. Patient recruitment and consent are performed by the clinical research coordinator. A standardised script is used to describe objectives, risks and benefits and details of the randomisation to eligible participants. The sample size required to carry out this study is 50. This figure is based on the number of potentially eligible UC patients at McMaster Children's Hospital (150) and Children's Hospital of Western Ontario (100) using recent census data from respective centres' IBD clinics. Twenty-five patients will be randomised to receive normal saline enemas (control; saline/polyethylene glycol 3350) and 25 patients to receive an FMT enema (intervention; $50 \mathrm{~g} / 150 \mathrm{~mL}, 10^{7}$ microbes/ $\mathrm{mL}$ of suspension in saline/polyethylene glycol 3350). This pilot study is not powered for any of the outcomes (table 1). Randomisation occurs according to a computer-generated block randomisation pattern (block size $=4$ participants). Eligible patients will be randomised 1:1 to receive FMT, or normal saline faecal enemas containing brown food colouring. Patients randomised to receive the normal saline placebo enema will be given an opportunity to enter an open-label phase of the study at completion, to receive the faecal microbial enema and re-enter the trial through a prospective observational design.

\section{Enema product}

Saline and faecal enemas are both obtained from Rebiotix, a biotechnology company from Minnesota, USA that distributes live, human-derived faecal microbial enemas (RBX2660). RBX2660 has received Health Canada Clinical Trials Application (CTA) and US Food and Drug Administration Investigational New Drug Application (IND) approvals for clinical trials inpatients with recurrent $C$. difficile infection. Enemas contain live, unprocessed human faecal microbiota or saline with polyethylene glycol preservative (placebo). Faecal microbial donors are screened, stools are tested for infectious pathogens, centrally prepared in opaque enema bags, retested prior to delivery and sent by Rebiotix to the investigator through preserved cold-chain delivery. Specifically, the samples are stored in $-80^{\circ} \mathrm{C}$ freezers at Rebiotix, Minnesota, USA with next-day, on-site delivery in styrofoam-insulated boxes containing ice packs. Frozen enemas are removed from the boxes and stored in the clinic fridge $\left(4^{\circ} \mathrm{C}\right)$ for up to 3 days. The opaque enema bags and enema tubing ensure that blinding is preserved to the study patient.

A study team member initially 'connects' the enema bag to its tubing. We add $1 \mathrm{~mL}$ of commercially available (Club House brand), food-grade food colouring to the normal saline enema bag (two drops red, three drops green and seven drops yellow) to confer a brown colour to the clear normal saline solution.

\section{Statistical analysis \\ Outcomes}

Subjects will have outcome measures performed at four time points (figure 1). In addition to study outcome measures, patients in each randomisation arm will receive usual medical care.

Feasibility will be assessed by the outcomes of patient eligibility, patient recruitment, patient retention, adherence to blood and stool sample collection times and adverse events or patient hospitalisations throughout the enema administration and follow-up period.

Bloodwork collection will be performed at the time of the FMT (weeks 0, 3 and 6), or at scheduled clinic visits (weeks 18 and 30). Standard paediatric UC bloodwork to monitor for systemic inflammation will be ordered: complete blood count, erythrocyte sedimentation rate, CRP, alanine transaminase, aspartate transaminase, alkaline phosphatase. Routine laboratory protocols and assays for obtaining and measuring samples will be followed. ${ }^{8}$

Clinical disease activity scores will be determined based on a history obtained at each clinical assessment (two times per week on weeks $0-6$; once on weeks 18 and 30), or over the telephone on non-clinical assessment days (weeks 12 and 24). The internationally validated PUCAI score will be used to objectively assess clinical disease activity. ${ }^{21}$

Stool samples will be collected for microbiome analysis and faecal calprotectin (weeks 0, 3, 6, 12, 18, 24 and 30) to assess patterns of bacterial community structure associated 
Table 1 Trial outcomes (feasibility and clinical outcomes)

\begin{tabular}{|c|c|c|c|c|}
\hline & Outcome & Measure & & Analysis \\
\hline \multirow{6}{*}{$\begin{array}{l}\text { Feasibility } \\
\text { outcomes }\end{array}$} & Participant recruitment & Recruitment/month & \multirow{6}{*}{$\begin{array}{l}\text { Cumulative } \\
\text { across all } \\
\text { study sites }\end{array}$} & \multirow{2}{*}{$\begin{array}{l}\geq 2 \text { participants/month } \\
\text { recruited and retained } \\
\text { for duration of study }\end{array}$} \\
\hline & Participant retention & Percent dropout post enrolment & & \\
\hline & Adverse events & $\begin{array}{l}\text { Hospitalisation or } \\
\uparrow P U C A I \geq 20 \times 2 \text { consecutive } \\
\text { measures }\end{array}$ & & $<10 \%$ of participants \\
\hline & Stool specimens & $\begin{array}{l}\text { Participants provides all required } \\
\text { stool samples }\end{array}$ & & $>90 \%$ of participants \\
\hline & Microbiome & $\begin{array}{l}\text { Microbiome analyses ( } 16 \mathrm{~s} \text { rRNA } \\
\text { profile, metagenomics) performed } \\
\text { for participant at all required time } \\
\text { points }\end{array}$ & & $>80 \%$ of participants \\
\hline & Week 30 Endoscopy & Endoscopy obtained & & $>10 \%$ of participants \\
\hline \multirow{7}{*}{$\begin{array}{l}\text { Clinical } \\
\text { outcomes }\end{array}$} & Clinical remission (6 weeks) & \multicolumn{2}{|l|}{$\mathrm{PUCAI} \leq 10$} & $\chi^{2}$ test \\
\hline & Clinical remission (30 weeks) & \multicolumn{2}{|l|}{$\mathrm{PUCAI} \leq 10$} & $\chi^{2}$ test \\
\hline & Clinical remission (6-30 weeks) & \multicolumn{2}{|l|}{ Sustained PUCAI $<10$} & $\chi^{2}$ test \\
\hline & Clinical improvement (6 weeks) & \multicolumn{2}{|l|}{$\downarrow \mathrm{PUCAI} \geq 15$} & $\chi^{2}$ test \\
\hline & Clinical improvement (6-30 weeks) & \multicolumn{2}{|l|}{ Sustained $\downarrow$ PUCAI $\geq 15$} & $\chi^{2}$ test \\
\hline & Biological improvement (6 weeks) & \multicolumn{2}{|l|}{$\downarrow$ C-reactive protein } & t-test \\
\hline & Biological improvement (6 weeks) & \multicolumn{2}{|l|}{$\downarrow$ faecal calprotectin } & t-test \\
\hline
\end{tabular}

PUCAI, Paediatric Ulcerative Colitis Activity Index.

with intestinal inflammation. Routine collection of stool specimens will be performed at home independently by patients. Stools unable to be submitted immediately may be kept in a sterile screw-capped sample container in a standard, home freezer until delivered to the laboratory. Microbiome analysis will be conducted through the laboratory of Dr M. Surette; bacterial community profiling of $16 \mathrm{~s}$ rRNA genes will be performed on part of each stool sample using 250 nucleotide paired end reads of the V3 (or V3V4) region using the MiSeq Illumina sequencer. ${ }^{26}$ Analysis will be performed using an in-house bioinformatics pipeline that generates clusters of operational taxonomic units, taxonomic assignment and various measures of alpha and beta diversity. If required at the bioinformatics stage, additional control microbial data will be obtained through Human Microbiome Project published databanks.

Imaging and endoscopic surveillance may be performed if clinically indicated.
Follow-up patient outcomes will be measured at weeks 12, 18, 24 and 30 after the faecal enema intervention (figure 1).

All primary and secondary outcomes will be assessed using both intention-to-treat and per-protocol. The differences in remission rates between the groups will be analysed with support from departmental statistical support personnel. Proportions and percentages will be reported to determine whether all feasibility outcomes were reached. Continuous outcomes will be compared using t-tests and categorical outcomes will be compared using $\chi^{2}$ tests. 95\% CIs and $\mathrm{p}$ values will be reported (table 1 ).

\section{Safety monitoring}

Study risks include enema delivery, and complications of FMT. Enema administration carries a low risk of proctitis, and FMT has been associated with $C$. difficile colitis, fever and infection. ${ }^{26}$ 
We will be obtaining FMT materials through a company called Rebiotix, a biotechnology firm located in Minnesota, USA. The faecal enema (RBX2660) prepared by Rebiotix has received Health Canada Clinical Trials Application (CTA) and US Food and Drug Administration Investigational New Drug Application (IND) approvals for clinical trials inpatients with recurrent $C$. difficile infection.

Donor qualification processes involve: potential donors completing a health and lifestyle questionnaire, providing blood and stool samples for analyses of (1) Blood-HIV, hepatitis A, B and C, syphilis and (2) Stool-C. difficile toxin B, Norovirus, Rotavirus, Adenovirus, Shigella, Salmonella, Campylobacter, Escherichia coli, Aeromonas, Plesiomonas, Yersinia, Shiga toxins, Giardia antigen, Cryptosporidium antigen, Cyclospora, Isospora, ova and parasites, Vancomycin-resistant enterococci, methicillin-resistant Staphylococcus, Vibrio and Listeria.

Donors are screened to confirm whether they are disease-free before donations begin. When donations begin, a sample of each donation from each donor is retained. Retained samples from each donor are pooled with other samples; from that, same donor and the pooled samples are subjected to repeat stool testing at approximately 45-day intervals to confirm continued donor health. Repeat donor blood testing is also performed at a minimum of 14 days after the last donation and again during the approximately 45-day collection interval. If the donor passes the repeat screening, drug product manufactured from donations collected within that 45-day period is released from quarantine. At the time of each donation, the donor completes a repeat questionnaire to confirm his/her continued health and absence of risky lifestyle behaviours. Rebiotix performs stool and blood testing approximately every 45 days.

Study participants will be monitored for signs of clinical deterioration. PUCAI scores will be measured at defined trial time points (figure 1). An increase in disease activity score of $\geq 20$, from the previously collected score, will be classified as 'disease progression'. ${ }^{21}$ Reassessment of PUCAI scoring will occur within 1 week and any further increase $\geq 20$ will remove the patient from the study for implementation of standard IBD management. Patients who contact study coordinators or clinic nurses reporting fever, or worsening vomiting, abdominal pain, rectal pain, diarrhoea or hematochezia will have PUCAI scores measured to evaluate for progression. All participants who experience an increase in PUCAI scores of $\geq 20$ at successive measurements, or admission to hospital for any reason, will be classified as having an adverse event, and unblinding may be performed to the treating clinician and/or study participant at that time.

A Data, Safety and Monitoring Committee (DSMC) has been assembled for the study at the investigators' discretion. The DSMC will perform an interval assessment of preliminary study data, approximately midway through the trial. The DSMC comprised experts in the fields of paediatric gastroenterology, paediatric IBD management and clinical trial statistical analyses, and will operate independently of the investigators and collaborators for the trial, and any sponsors. The terms of the DSMC will be defined at the initial meeting, which has not yet occurred. The DSMC was not assembled earlier, due to the Research Ethics Board at the primary site determining that a DSMC would not be necessary for the trial to commence.

\section{ETHICS AND DISSEMINATION \\ Ethics}

The study will be conducted in accordance with the protocol, the Helsinki declaration, and the Canadian Tri-Council Policy Statement on research ethics. A Health Canada no-objection letter to perform FMT for the purpose of the trial was obtained, and local research ethics board (REB) approval was obtained for McMaster Children's Hospital and Children's Hospital of Western Ontario. All amendments to the trial protocol will be obtained through approval by the local REB and updated through the clinical trials registry (ClinicalTrials.gov). Research personnel will approach all potentially eligible patients who fulfil eligibility criteria for consent. All patients must sign a consent form to participate in the trial.

\section{Data sharing}

Anonymised data about patient outcomes will be shared with Rebiotix for the assessment of RBX2660 (faecal enema) efficacy. The information shared will be limited to: (1) patients' age, height and weight, (2) patients' medication history (previous, and ongoing), (3) date enema was given, volume administered any reported adverse effects of delivery and (4) PUCAI scores, results of bloodwork and faecal calprotectin.

In addition, a deidentified stool sample will be sent to Rebiotix. These will be collected along with standard stool samples per study protocol (figure 1).

\section{Follow-up}

Results of this pilot will inform several future research goals. Pilot data will be used to inform a definitive multicentre randomised-controlled trial using a larger paediatric patient population. Data will be used to support additional research funding for a multicentre trial through a Canadian Institute of Health Research operating grant. Our data may also support further cross-over studies between human and mouse models to ascertain host-microbial influences underlying observed changes.

\section{Reporting}

Results of the pilot will be reported to key stakeholders. Pilot data will be shared locally with clinical and basic science division members. Results will be shared through presentations at regional hospitals to encourage participation in future multicentre trials. Pilot data will also be submitted to international conferences and published in peer-review journals. 


\section{FUTURE DIRECTIONS}

On the basis of the results of this study, future doubleblind, randomised, placebo-controlled trials of FMT in paediatric IBD may involve:

\section{Primary outcomes}

- Evaluation of efficacy of FMT at inducing clinical remission in new-onset UC or Crohn's disease.

- Evaluation of efficacy of multiple, periodically administered FMT at maintaining clinical remission (prevention of relapse).

\section{Secondary outcomes}

- Evaluation of efficacy of FMT at inducing long-term mucosal healing.

- Evaluation of change in faecal bacterial community structures of patients receiving FMT.

\section{DISCUSSION}

Increasing prevalence of UC worldwide, particularly in Canada, and diagnosis in increasingly younger populations necessitate the need for novel treatment approaches. ${ }^{27}$ Currently, this trial is open to paediatric UC and patients with IBD-U experiencing symptoms of clinical or mucosal inflammation, who would like to abstain from escalation of therapy or surgical interventions.

A previous randomised-controlled trial for FMT in adult UC demonstrated a statistically significant benefit of FMT $(9 / 38,24 \%$ vs $2 / 37,5 \%)$ at inducing UC remission, with no significant difference in adverse events between the groups. ${ }^{20}$ A second positive randomised-controlled trial for FMT in adult UC was recently published, showing statistically significant benefits of FMT versus normal saline placebo at inducing: steroid-free clinical remission $(18 / 41,44 \%$ vs $8 / 40,23 \%)$, clinical response (22/41, $54 \%$ vs $9 / 40,23 \%)$ and endoscopic response $(13 / 41$, $32 \%$ vs $4 / 40,10 \%) .^{28}$

This study will use a similar 6 -week approach, with the two time per week enema administrations instead of one time per week. We will use a similar normal saline enema placebo. Our trial will also involve a greater diversity of anonymous, non-household donors, to decrease the likelihood of observing a donor-dependent outcome. Primary outcomes of this pilot study will be feasibility measures of the reported protocol, and secondary outcomes will include subjective and objective measures of clinical and mucosal healing of UC/IBD-U over the enema administration and follow-up period.

This trial will provide preliminary evidence for the use of FMT in a paediatric UC population. Our results will be informative for future, larger population size, doubleblinded randomised-controlled trial in paediatric UC, which may look into further analysing the efficacy of FMT in inducing remission and optimise dosage.

Acknowledgements Christine Lee provided substantial contributions to design and regulatory approval of the trial, and acquisition of trial data. Paul Moayyedi provided substantial contributions to design of the trial.Michael Surette provided substantial contributions to design of the trial, acquisition and analysis of preliminary trial data. Waliul Khan provided substantial contributions to acquisition and analysis of preliminary trial data.

Contributors NP: substantial contributions to conception and design of trial, acquisition of trial data; drafting of protocol; final approval of the protocol to be published; agreement to be accountable for all aspects of the work. JP: substantial contributions to design of trial, acquisition of trial data; drafting of protocol; final approval of the protocol to be published; agreement to be accountable for all aspects of the work.

Funding This work was supported by (1) Hamilton Health Sciences New Investigator Fund (2015, Spring) (http://www.hhsresearchadmin.ca) and (2) Innovation Fund of the Alternative Funding Plan for the Academic Health Sciences Centres of Ontario (HAH-17-002) (http://www.hahso.ca/).

Competing interests None declared.

Patient consent Guardian consent obtained.

Ethics approval Hamilton Integrated Research Ethics Board.

Provenance and peer review Not commissioned; externally peer reviewed.

Open Access This is an Open Access article distributed in accordance with the Creative Commons Attribution Non Commercial (CC BY-NC 4.0) license, which permits others to distribute, remix, adapt, build upon this work non-commercially, and license their derivative works on different terms, provided the original work is properly cited and the use is non-commercial. See: http://creativecommons.org/ licenses/by-nc/4.0/

(C) Article author(s) (or their employer(s) unless otherwise stated in the text of the article) 2017. All rights reserved. No commercial use is permitted unless otherwise expressly granted.

\section{REFERENCES}

1. Rocchi A, Benchimol El, Bernstein CN, et al. Inflammatory bowel disease: a canadian burden of illness review. Can J Gastroenterol 2012;26:811-7.

2. Kappelman MD, Rifas-Shiman SL, Kleinman $\mathrm{K}$, et al. The prevalence and geographic distribution of crohn's disease and ulcerative colitis in the United States. Clin Gastroenterol Hepatol 2007;5:1424-9.

3. Benchimol El, Bernstein CN, Bitton A, et al. Trends in epidemiology of pediatric inflammatory bowel disease in Canada: Distributed network analysis of multiple population-based provincial health administrative databases. Am J Gastroenterol 2017.

4. El Mouzan MI, Al Mofarreh MA, Saadah OI, et al. Impact of pediatric inflammatory bowel disease on linear growth: data from a national cohort study in Saudi Arabia. Saudi J Gastroenterol 2016;22:396-8.

5. Sanderson IR. Growth problems in children with IBD. Nat Rev Gastroenterol Hepatol 2014;11.

6. Fuller-Thomson E, Sulman J. Depression and inflammatory bowel disease: findings from two nationally representative canadian surveys. Inflamm Bowel Dis 2006;12:697-707.

7. de Ridder L, Turner D, Wilson DC, et al. Malignancy and mortality in pediatric patients with inflammatory bowel disease: a multinational study from the porto pediatric IBD group. Inflamm Bowel Dis 2014;20:291-300.

8. Talley NJ, Abreu MT, Achkar JP, et al. American College of Gastroenterology IBD Task Force. An evidence-based systematic review on medical therapies for inflammatory bowel disease. $A m ~ J$ Gastroenterol 2011;106:S2-S25.

9. Torres J, Danese S, Colombel JF. New therapeutic avenues in ulcerative colitis: thinking out of the box. Gut 2013;62:1642-52.

10. Kassam Z, Lee CH, Yuan Y, et al. Fecal Microbiota transplantation for Clostridium difficile infection: systematic review and meta-analysis. Am J Gastroenterol 2013;108:500-8.

11. Michail S, Durbin M, Turner D, et al. Alterations in the gut microbiome of children with severe ulcerative colitis. Inflamm Bowel Dis 2012;18:1799-808.

12. Nell S, Suerbaum S, Josenhans $C$. The impact of the Microbiota on the pathogenesis of IBD: lessons from mouse infection models. Nat Rev Microbiol 2010;8:564-77.

13. Kunde S, Pham A, Bonczyk S, et al. Safety, tolerability, and clinical response after fecal transplantation in children and young adults with ulcerative colitis. J Pediatr Gastroenterol Nutr 2013;56:597-601.

14. Kellermayer R, Nagy-Szakal D, Harris RA, et al. Serial fecal Microbiota transplantation alters mucosal gene expression in pediatric ulcerative colitis. Am J Gastroenterol 2015;110:604-6. 
15. Suskind DL, Brittnacher MJ, Wahbeh G, et al. Fecal microbial transplant effect on clinical outcomes and fecal microbiome in active crohn's disease. Inflamm Bowel Dis 2015;21:556-63.

16. Suskind DL, Singh N, Nielson $\mathrm{H}$, et al. Fecal microbial transplant via nasogastric tube for active pediatric ulcerative colitis. $J$ Pediatr Gastroenterol Nutr 2015;60:27-9.

17. Vandenplas Y, Veereman G, van der Werff Ten Bosch J, et al. Fecal microbial transplantation in early onset colitis: caution advised. $J$ Pediatr Gastroenterol Nutr 2015;61:e12-14.

18. Shimizu H, Arai K, Abe J, Nakabayashi K, et al. Repeated fecal microbiota transplantation in a child with ulcerative colitis. Pediatr Int 2016;58:781-5.

19. Kumagai H, Yokoyama K, Imagawa T, et al. Failure of fecal Microbiota transplantation in a Three-Year-Old child with severe refractory ulcerative colitis. Pediatr Gastroenterol Hepatol Nutr 2016;19:214-20.

20. Moayyedi P, Surette MG, Kim PT, et al. Fecal Microbiota transplantation induces remission in patients with active ulcerative colitis in a Randomized Controlled Trial. Gastroenterology 2015;149:102-9.

21. Turner D, Otley AR, Mack D, et al. Development, validation, and evaluation of a pediatric ulcerative colitis activity index: a prospective multicenter study. Gastroenterology 2007;133:423-32.
22. Turner D, Mack DR, Hyams J, et al. C-reactive protein (CRP), erythrocyte sedimentation rate (ESR) or both? A systematic evaluation in pediatric ulcerative colitis. $J$ Crohns Colitis 2011;5:423-9.

23. Turner D, Levine A, Escher JC, et al. Management of pediatric ulcerative colitis: joint ECCO and ESPGHAN evidencebased consensus guidelines. J Pediatr Gastroenterol Nutr 2012;55:340-61.

24. Snapper SB. Very-Early-Onset inflammatory bowel disease. Gastroenterol Hepatol 2015;11:554-6.

25. Turner D, Muise AM. Very Early Onset IBD: how very Different 'on Average'? J Crohns Colitis 2017;11:jjw217-2.

26. Kelly CR, Ihunnah C, Fischer M, et al. Fecal Microbiota transplant for treatment of Clostridium difficile infection in immunocompromised patients. Am J Gastroenterol 2014;109:1065-71.

27. Benchimol El, Guttmann A, Griffiths AM, et al. Increasing incidence of paediatric inflammatory bowel disease in Ontario, Canada: evidence from health administrative data. Gut 2009;58:1490-7.

28. Paramsothy S, Kamm MA, Kaakoush NO, et al. Multidonor intensive faecal Microbiota transplantation for active ulcerative colitis: a randomised placebo-controlled trial. Lancet 2017;389:1218-28. 